\title{
Inversão de Velocidades Intervalares: Aplicação em Modelo da Área do Pré-sal
}

Thaís G. Santana, IGEO/UFBA \& Amin Bassrei, CPGG/IGEO/UFBA

Copyright 2014, SBGf - Sociedade Brasileira de Geofísica

Este texto foi preparado para a apresentação no VI Simpósio Brasileiro de Geofísica, Porto Alegre, 14 a 16 de outubro de 2014. Seu conteúdo foi revisado pelo Comitê Técnico do VI SimBGf, mas não necessariamente representa a opinião da SBGf ou de seus associados. É proibida a reprodução total ou parcial deste material para propósitos comerciais sem prévia autorização da SBGf.

\section{Resumo}

A determinação de velocidades intervalares é um parâmetro fundamental buscado no processamento sísmico, o que pode feito com a utilização da fórmula de Dix. Nesse trabalho a distribuição de velocidades intervalares é estabelecida como problema inverso, que por sua vez é resolvido pela decomposição por valores singulares (SVD) e pela entropia relativa mínima (MRE). Em ambos os casos é possível a incorporação de informação a priori. Foram realizadas simulações em um modelo sintético bidimensional, inspirado numa seção da área do Pré-sal da Bacia de Santos. A seção bidimensional foi decomposta em 1000 perfis unidimensionais. Para cada perfil é foi realizada uma inversão, e as 1000 distribuições de velocidades intervalares estimadas fora agrupadas formando uma distribuição bidimensional de velocidade. O problema inverso é classificado como subdeterminado uma vez que para cada inversão com 1000 incógnitas são utilizadas, a depender do caso, apenas 20 ou 50 equações. Os resultados foram satisfatórios para o SVD e o MRE, sendo $O$ MRE ligeiramente superior em algumas simulações.

\section{Introdução}

Os métodos sísmicos utilizam a teoria de propagação de ondas elásticas no interior da Terra, com o objetivo de adquirir imagens da subsuperfície para fins de exploração de alvos comerciais. Tais métodos são os mais importantes para a indústria de petróleo, com destaque para a sísmica de reflexão.

Neste trabalho foram utilizadas diferentes metodologias de obtenção da distribuição de velocidades intervalares com aplicações na área do Pré-sal. A velocidade intervalar é a velocidade de propagação do pulso sísmico em um dado meio ou camada geológica, e o seu conhecimento é necessário em diferentes etapas do processamento sísmico, como por exemplo a migração sísmica. Portanto, a estimativa precisa das velocidades intervalares permite caracterizar o meio analisado, além de garantir resultados de maior confiabilidade quanto à qualidade da imagem obtida.

O modelo geológico utilizado nesse trabalho é relativamente complexo, assim como acontece com a maioria dos modelos do mundo real. Consequentemente, a fórmula de Dix não é adequada nessas situações, já que os mesmos apresentam refletores curvos e/ou camadas com mergulho. Uma alternativa é tratar a obtenção do campo de velocidades intervalares como um problema inverso a ser resolvido. A aplicação da formulação de problemas inversos, usando decomposição por valores singulares (SVD) e entropia relativa mínima (MRE), tem como objetivo obter resolver problemas mal-postos.

Iremos classificar a abordagem da inversa generalizada, cuja implementação numérica é efetuada pelo SVD como determinística. Por outro lado o MRE é classificado como probabilístico. Essas duas abordagens permitem a incorporação de informação a priori, o que não é possível quando se utiliza a fórmula de Dix. A incorporação da informação a priori é mais crucial em problemas inversos subdeterminados, onde o número de incógnitas é superior ao número de equações.

Para validar suas utilizações, essas técnicas foram aplicadas em dados sintéticos unidimensionais, utilizando rotinas FORTRAN que foram desenvolvidas no CPGG/UFBA para a determinação as velocidades intervalares do meio. Posteriormente, o mesmo foi feito com dados sintéticos bidimensionais simulando uma área do Pré-sal, baseados no trabalho de Assine et al. (2008).

\section{Metodologia/ Problema Investigado}

A velocidade RMS pode ser calculada usando a equação:

$$
\mathrm{V}_{\mathrm{RMS}}^{2}(\mathrm{k})=\frac{\sum_{\mathrm{k}=1}^{\mathrm{n}} \mathrm{v}_{\mathrm{k}}^{2} \mathrm{t}_{\mathrm{k}}}{\sum_{\mathrm{k}=1}^{\mathrm{n}} \mathrm{t}_{\mathrm{k}}} .
$$

A inversão da equação (1) é chamada de e a fórmula de Dix:

$$
v_{n}^{2}=\frac{V_{R M S, n}^{2} T_{0, n}-V_{R M S, n-1}^{2} T_{0, n-1}}{T_{0, n}-T_{0, n-1}},
$$

onde:

- $\mathrm{v}_{\mathrm{n}}$ é a velocidade intervalar na camada $\mathrm{n}$;

- $\mathrm{T}_{0, \mathrm{n}}$ é o tempo duplo de trânsito da origem até a interface $n$;

- $V_{R M S, n}$ é a velocidade RMS da interface $n$.

$\mathrm{Na}$ formulação do problema inverso pelo MRE, os dados de entrada, ou seja, o vetor dado da inversão são o 
produto dos quadrados das velocidades RMS pelos seus respectivos tempos:

$$
d_{j}=T_{j} V_{R M S}^{2}\left(T_{j}\right), \quad j=1, \ldots, M .
$$

No MRE é necessária uma estimativa inicial do modelo $\mathrm{m}_{\mathrm{p}}$ como outro dado de entrada:

$$
\mathrm{m}_{\mathrm{p}}=\mathrm{v}_{\mathrm{i}}^{2}, \quad \mathrm{i}=1, \ldots, \mathrm{N}
$$

A solução pelo MRE é a estimativa posterior do modelo $\mathrm{m}_{\mathrm{i}}$ :

$$
\mathrm{m}_{\mathrm{i}}=\mathrm{v}_{\mathrm{i}}^{2}, \quad \mathrm{i}=1, \ldots, \mathrm{N},
$$

onde $v_{i}$ é a velocidade intervalar da i-ésima camada.

Uma matriz $\mathrm{G}_{\mathrm{MxN}}$ retangular de posto $\mathrm{K}$ pode ser decomposta pelo SVD como (Menke, 1984)

$$
\mathrm{G}=\mathrm{U} \Sigma \mathrm{V}^{\mathrm{T}}
$$

onde:

- U é a matriz MxM dos autovetores ortonormalizados de $\mathrm{GG}^{\mathrm{T}}$;

- $\Sigma$ é a matriz diagonal MxN com a raiz quadrada dos autovalores da matriz $\mathrm{G}^{\mathrm{T}} \mathrm{G}$; e

- V é a matriz NxN dos autovetores ortonormalizados da matriz $\mathrm{G}^{\mathrm{T}} \mathrm{G}$.

Neste caso, a inversa generalizada ou pseudo-inversa, representada por $\mathrm{G}^{+}$, é uma matriz $\mathrm{NxM}$ dada por:

$$
\mathrm{G}^{+}=\mathrm{V} \Sigma^{+} \mathrm{U}^{\mathrm{T}}
$$

Esta matriz pseudo-inversa é única e para tanto deve atender as condições de Penrose.

A abordagem determinística utiliza o método dos mínimos quadrados (MMQ) para determinar a solução, cuja função objetivo é dada por:

$$
\phi(\mathrm{m})=\mathbf{m}^{\mathrm{T}} \mathbf{m}+\mathbf{t}^{\mathrm{T}}(\mathbf{d}-\mathrm{Gm}),
$$

onde t é o vetor dos multiplicadores de Lagrange.

A solução da função objetivo acima é:

$$
\mathbf{m}=G^{\mathrm{T}}\left(\mathrm{GG}^{\mathrm{T}}\right)^{+} \mathbf{d} \text {. }
$$

O processo de inversão através do SVD pode incorporar informação prévia $\mathbf{m}_{0}$. Se modificarmos a função objetivo para

$$
\phi(\mathrm{m})=\left(\mathbf{m}-\mathbf{m}_{0}\right)^{2}+2 \mathbf{t}^{\mathrm{T}}(\mathbf{d}-\mathrm{Gm}),
$$

chegaremos à seguinte solução:

$$
\mathbf{m}=G^{\mathrm{T}}\left(\mathrm{GG}^{\mathrm{T}}\right)^{+}\left(\mathbf{d}-\mathrm{Gm}_{0}\right)+\mathbf{m}_{0} .
$$

Para o método MRE utilizamos a abordagem da teoria contínua da inversão, onde lidamos com a equação integral de Fredholm, que representa o tipo de problema inverso que abordamos (Ulrych et al., 1990):

$$
d_{j}=\int_{a}^{b} H_{j}(u) m(u) d u,
$$

onde:

- $\mathrm{d}_{\mathrm{j}}$ representam o conjunto discreto de parâmetros de dados;

- $\mathrm{H}_{\mathrm{j}}(\mathrm{u})$ são as funções que forma o núcleo ou a matriz da inversão;

- m(u) é a função que representa os parâmetros de modelo.

Nosso objetivo é obter uma estimativa final de $\mathrm{m}$, denotado por $\widetilde{m}$ que satisfaz a equação integral acima e é a solução do problema de minimização. Após a minimização da função objetivo juntamente com as restrições incorporadas, a seguinte expressão é obtida:

$$
\widetilde{\mathrm{m}}(\mathrm{i})=\frac{1}{\frac{1}{\mathrm{~s}(\mathrm{u})}+\sum_{\mathrm{j}=1}^{\mathrm{M}} \lambda_{\mathrm{j}} \mathrm{H}_{\mathrm{ji}}},
$$

- s(u) é a estimativa inicial do modelo a ser introduzida na inversão;

- $\mathrm{H}_{\mathrm{ji}}$ são os elementos da matriz e equivalem às funções $\mathrm{H}_{\mathrm{j}}(\mathrm{u})$;

- $\lambda_{j}$ são os multiplicadores de Lagrange definidos a partir do problema de minimização com restrições, e calculados com o método de Newton-Rapson para um sistema de equações não lineares.

O desempenho dos dois métodos (SVD e MRE) foi avaliado usando o critério de erro RMS relativo percentual $\left(\epsilon_{\mathrm{RMS}}\right)$ dos parâmetros de dados e de modelos que são expressos, respectivamente, por:

$$
\epsilon_{\mathrm{d}}=\frac{\sqrt{\sum_{\mathrm{i}=1}^{\mathrm{M}} \sum_{\mathrm{j}=1}^{\mathrm{N}}\left(\mathrm{d}_{\mathrm{i}, \mathrm{j}}^{\mathrm{obs}}-\mathrm{d}_{\mathrm{i}, \mathrm{j}}^{\mathrm{cal}}\right)^{2}}}{\sqrt{\sum_{\mathrm{i}=1}^{\mathrm{M}} \sum_{\mathrm{j}=1}^{\mathrm{N}}\left(\mathrm{d}_{\mathrm{i}, \mathrm{j}}^{\mathrm{obs}}\right)^{2}}} \times 100 \%,
$$

e,

- $\mathrm{d}^{\text {obs }}$ é vetor de parâmetros de dados observado; 
- $\mathrm{d}^{\text {cal }}$ é vetor de parâmetros de dados calculado;

- ver é o vetor de velocidades verdadeiras;

- $v^{\text {est }}$ é o vetor de velocidades estimadas.

Nas expressões acima M representa o número de traços. Para o estimador relacionado aos parâmetros de modelo $\mathrm{N}$ representa o número de camadas ou velocidades intervalares. Já para o estimador relacionado aos parâmetros de dado $\mathrm{N}$ representa $\mathrm{o}$ número de pares (velocidade RMS \& tempo de reflexão).

\section{Resultados}

As metodologias propostas na obtenção do campo de velocidades intervalares foram aplicadas a um modelo sintético bidimensional (Figura 1) composto por camadas planas com incidência vertical.

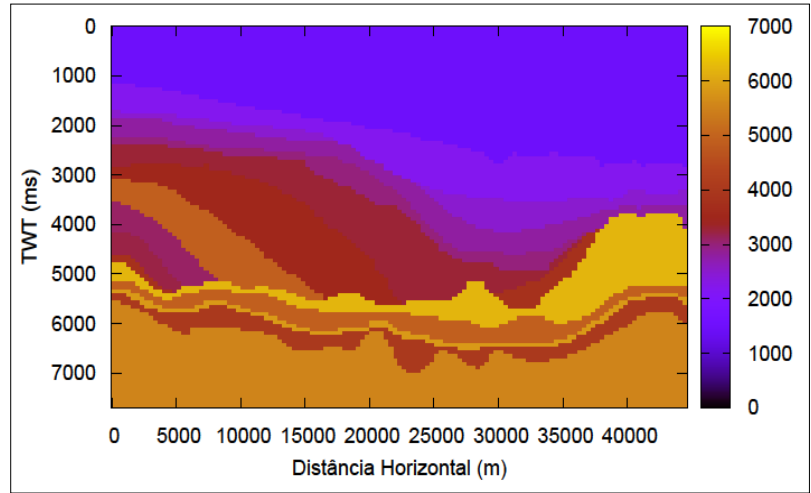

Figura 1 - Modelo bidimensional da área do Pré-sal. O eixo vertical representa o tempo de reflexão em $m s$, e o eixo horizontal a distância em $m$. A barra de corres indica as velocidades intervalares verdadeiras em $\mathrm{m} / \mathrm{s}$.

Esse modelo é uma situação típica do Pré-sal, na Bacia de Santos (Figura 2), apresentando uma lâmina de água com $2000 \mathrm{~m}$ em média, e com velocidade de $1500 \mathrm{~m} / \mathrm{s}$. O primeiro refletor do modelo tem velocidade de $2200 \mathrm{~m} / \mathrm{s}$. Os refletores desse modelo são mergulhantes, representado o topo da primeira camada até a base da última com velocidades variando de 2200 a $6200 \mathrm{~m} / \mathrm{s}$. O tempo de reflexão varia de 0 até $7.770 \mathrm{~ms}$, e a distância horizontal variando entre 0 e $44640 \mathrm{~m}$.

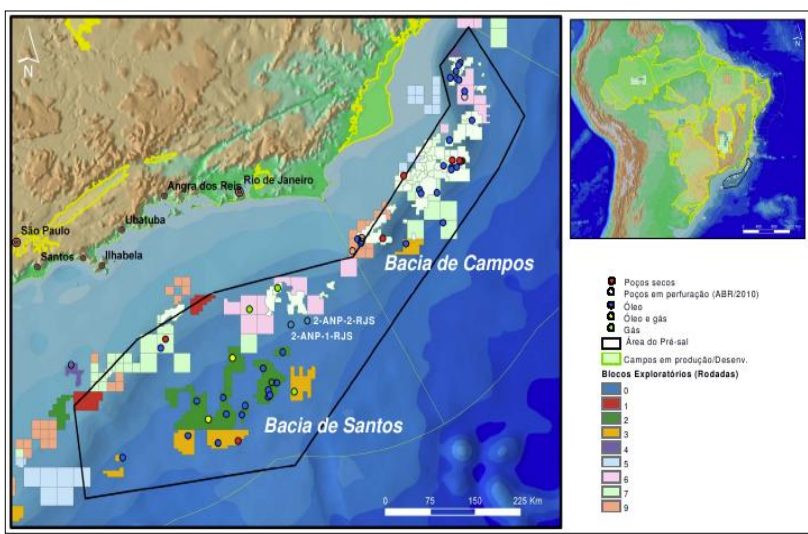

Figura 2 - Mapa das bacias brasileiras com camada de Pré-sal. Figura extraída da Agência Nacional de Petróleo, Gás Natural e Biocombustíveis (www.anp.gov.br).

Inicialmente, a fórmula de Dix foi aplicada diretamente aos dados de velocidade RMS, o que chamamos de abordagem clássica. As velocidades intervalares, obtidas pela fórmula de Dix, foram posteriormente comparadas com os resultados obtidos pela inversão pelo SVD e pela inversão pelo MRE.

O modelo bidimensional tem 1000 traços unidimensionais. Para traço considera um modelo de camadas plano-paralelas com 1000 camadas, sendo um valor de velocidade intervalar associado a cada camada. Cada inversão resulta numa saída com 1000 velocidades intervalares. Porém, o número de parâmetros de dados é, conforme o caso, apenas 20 ou 50 , ou seja, 20 ou 50 pares de velocidade RMS e tempo de reflexão associado à velocidade RMS. Desse modo o problema inverso é classificado como subdeterminado.

Para a configuração com 50 equações, o primeiro valor de velocidade RMS está associado ao pacote sedimentar das camadas 1 a 20, o segundo valor de velocidade RMS está associado ao pacote sedimentar das camadas 1 a 40, e assim por diante. Já na configuração com 20 equações, o primeiro valor de velocidade RMS está associado ao pacote sedimentar das camadas 1 a 50 , o segundo valor de velocidade RMS está associado ao pacote sedimentar das camadas 1 a 100, e assim por diante. A Figura 3 mostra as duas distribuições de velocidades. 


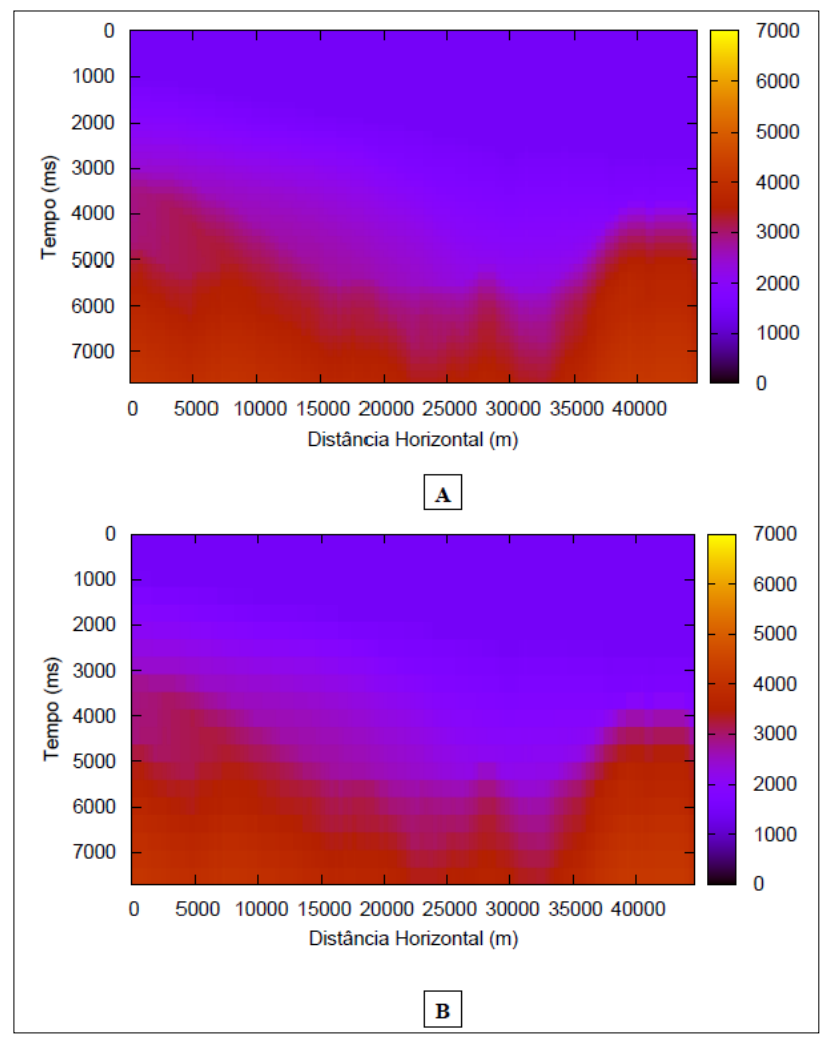

Figura 3 - Distribuição de velocidades RMS: (A) 50 velocidades e (B) 20 velocidades.

A Figura 4 mostra o campo de velocidades intervalares obtidas pelo SVD, sendo a Figura $4(\mathrm{~A})$ para 50 velocidades RMS e a Figura 4(C) para 20 velocidades RMS. Em ambos os casos o resultado do SVD foi igual à distribuição intervalares obtida pela fórmula de Dix. Para 0 caso de 50 velocidades RMS o erro foi $\epsilon_{\mathrm{dix}}=\epsilon_{\mathrm{SVD}}=$ $5.87 \%$ ). Já para 20 velocidades RMS o erro foi $\epsilon_{\mathrm{dix}}=$ $\epsilon_{\mathrm{SVD}}=9.22 \%$. Os resultados podem são considerados bons e consistentes. É possível obter a imagem residual, isto é, a imagem das velocidades estimadas subtraída das distribuições de velocidades verdadeiras, que está mostrada na Figura 4(B) para 50 velocidades RMS e na Figura 4(D) para 20 velocidades RMS.

A seguir foram utilizadas diversas informações a priori. Dois casos podem ser vistos na Figura 5, onde a velocidade a priori é constante na Figura $5(\mathrm{~A})$ e linearmente crescente na Figura 5(B).

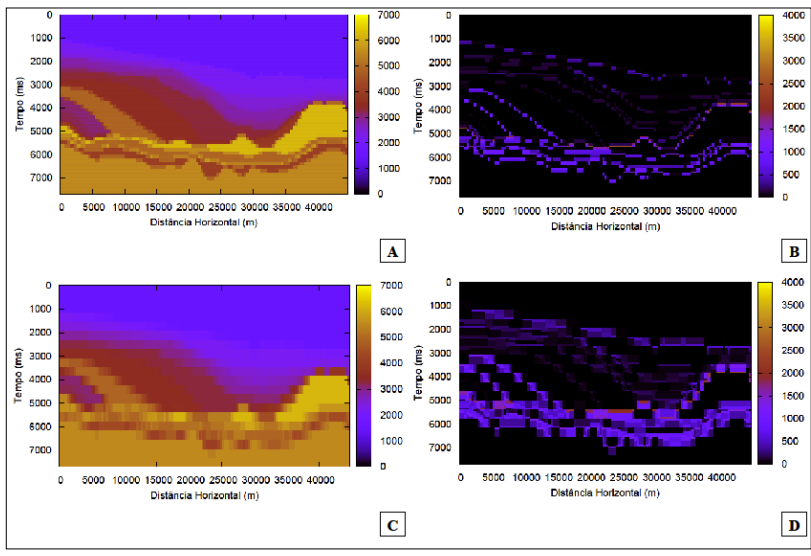

Figura 4 - (A) e (C) Distribuições de velocidades intervalares obtidos pelo SVD, respectivamente com 50 e 20 velocidades RMS, sem informação a priori. (B) e (D) Distribuições residuais entre as velocidades intervalares verdadeiras e as estimadas pelo SVD com, respectivamente, 50 e 20 velocidades RMS.

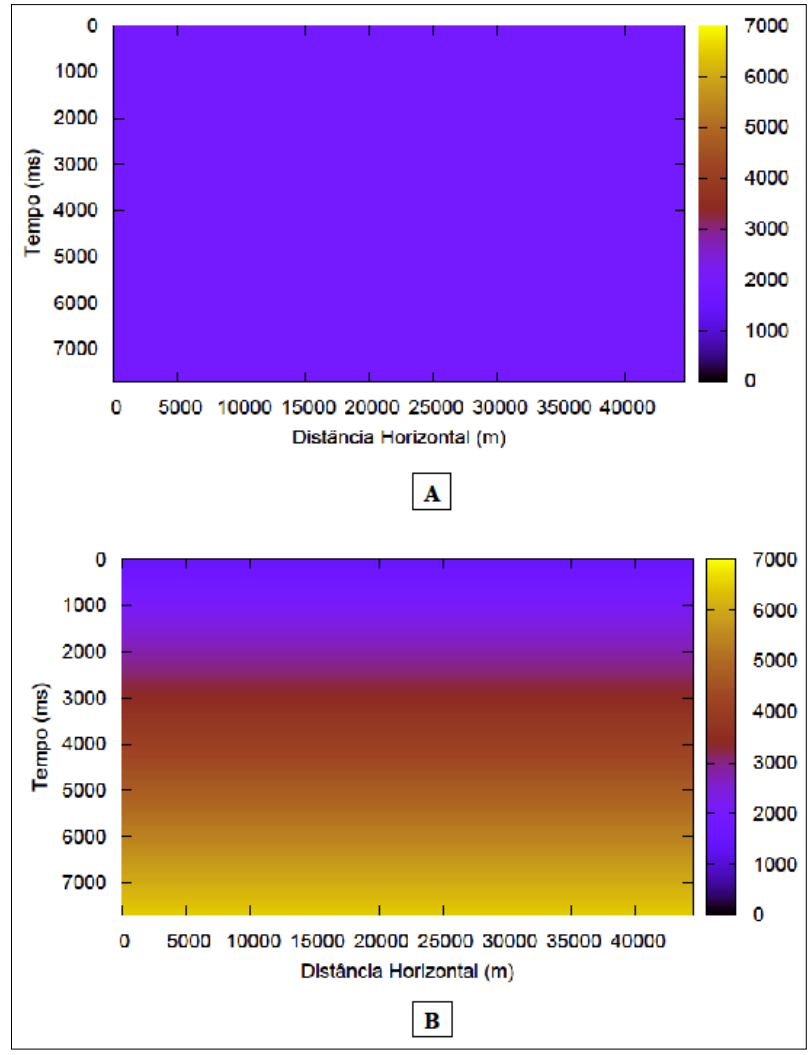

Figura 5 - Velocidades intervalares a priori, sendo em (A) constante e igual $2000 \mathrm{~m} / \mathrm{s}$ e em (B) linearmente crescente entre 1500 e $6500 \mathrm{~m} / \mathrm{s}$. 
Os resultados com a utilização a priori mostrados na Figura 5 podem ser vistos na Figura 6 para o caso de 50 velocidades RMS e na Figura 7 para o caso de 20 velocidades RMS. De um lado é óbvia a superioridade da inversão do sistema com 50 dados de entrada (Figura 6) em relação ao de 20 (Figura 7). Por outro lado, não se percebe uma superioridade visual do resultado que utiliza informação a priori linearmente crescente em relação ao resultado que utiliza o a priori constante.
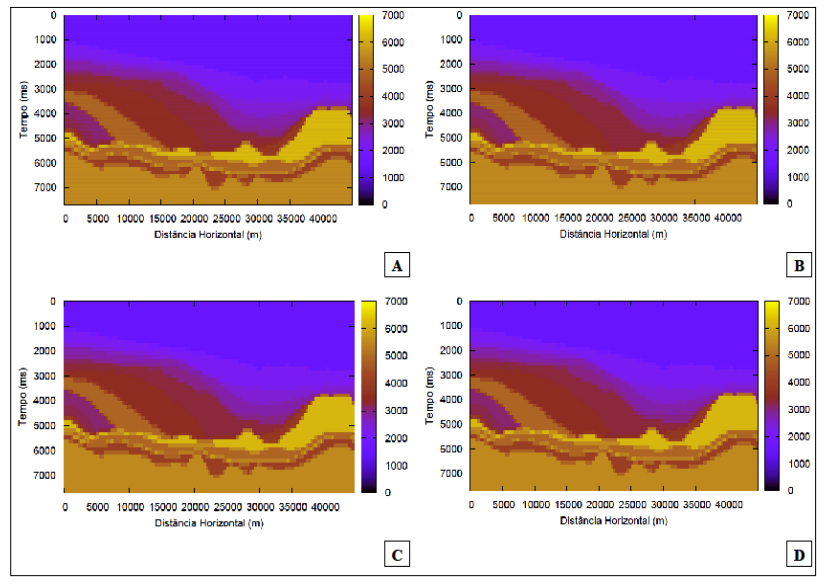

Figura 6 - Velocidades intervalares estimadas com 50 velocidades RMS: pelo método SVD em $(A)$ e $(C)$, e MRE em (B) e (D). Os resultados (A) e (B) utilizam informação a priori constante, enquanto (C) e (D) utilizam informacão a priori linearmente crescente.
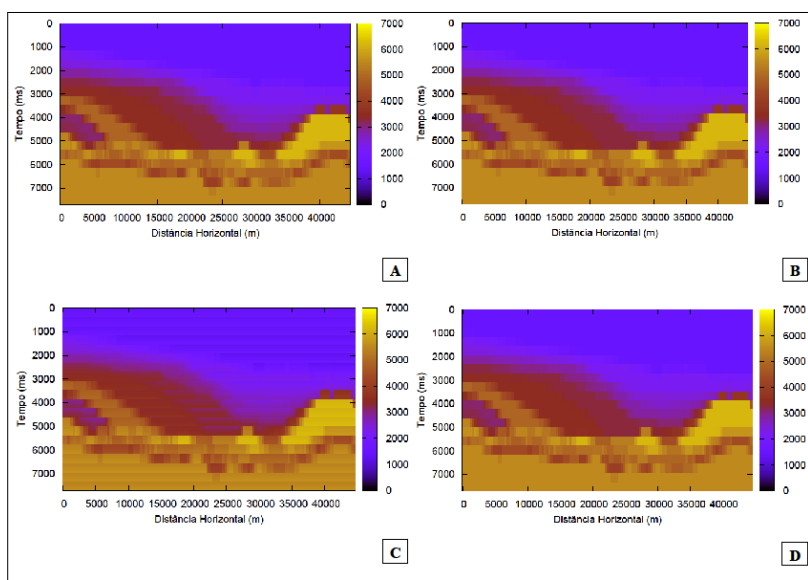

Figura 7 - Velocidades intervalares estimadas com 20 velocidades RMS: pelo método SVD em $(A)$ e $(C)$, e MRE em (B) e (D). Os resultados (A) e (B) utilizam informação a priori constante, enquanto (C) e (D) utilizam informação a priori linearmente crescente.
Para uma comparação quantitativa entre os dois métodos, foram geradas imagens residuais, assim como tabelas de resultados. A Figura 8 mostra as imagens residuais para as simulações com 50 velocidades RMS e a Figura 9 para o caso de 20 velocidades RMS. Os desvios são quantitativamente pequenos, representados pela coloração violeta, o que equivale a valores sempre inferiores a $1000 \mathrm{~m} / \mathrm{s}$. A cor preta por seu turno significa que a inversão foi bem sucedida nessa região do modelo.

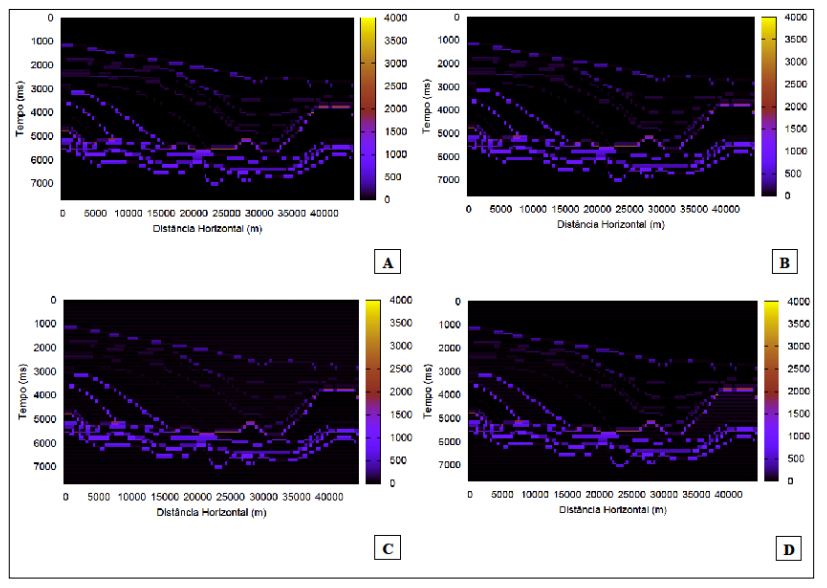

Figura 8 - Imagens residuais de velocidades intervalares estimadas com 50 velocidades RMS: pelo método SVD em (A) e (C), e MRE em (B) e (D). Os resultados $(A)$ e $(B)$ utilizam informação a priori constante, enquanto (C) e (D) utilizam informação a priori linearmente crescente.

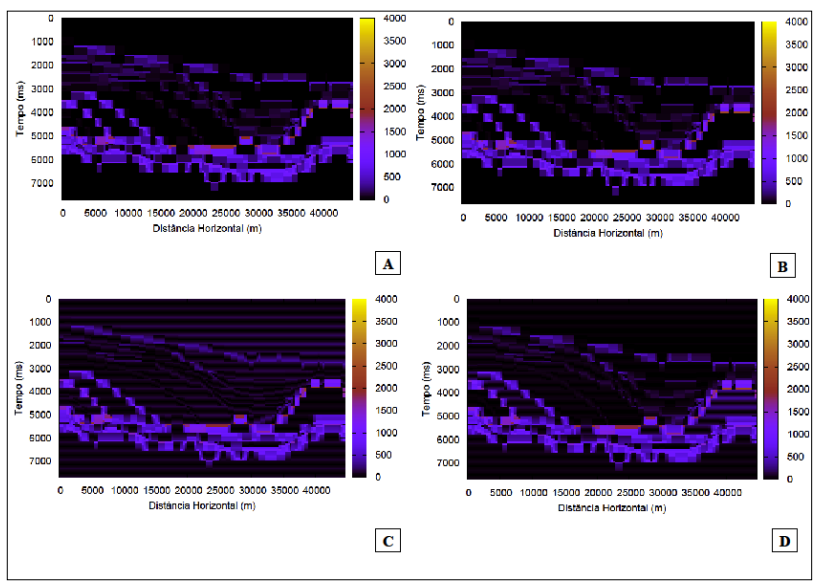

Figura 9 - Imagens residuais de velocidades intervalares estimadas com 20 velocidades RMS: pelo método SVD em (A) e (C), e MRE em (B) e (D). Os resultados $(A)$ e $(B)$ utilizam informação a priori constante, enquanto (C) e (D) utilizam informação a priori linearmente crescente. 
InVersão de Velocidades Intervalares: Aplicação em Modelo Sísmico da Área do Pré-Sal

Os erros das diversas simulações realizadas estão listados na Tabela 1 para o SVD e na Tabela 2 para o MRE. No caso do SVD a incorporação da informação a priori constante não melhora os resultados, e para a informação a priori linearmente crescente o erro diminui um pouco. Para o MRE, o resultado com a utilização de informação a priori é um pouco melhor que o SVD. Notese o erro da informação a priori, isto é, o erro RMS entre a distribuição de velocidades verdadeiras e 0 da informação a priori é $57.37 \%$ para o a priori constante e $24.89 \%$ para o a priori linearmente crescente.

\begin{tabular}{|c|c|c|c|}
\hline $\begin{array}{c}\text { Velocidades } \\
\text { RMS }\end{array}$ & A Priori & $\epsilon_{\mathrm{V}}(\%)$ & $\epsilon_{\mathrm{d}}(\%)$ \\
\hline \multirow{5}{*}{50} & $\begin{array}{c}\text { Sem } \\
\text { a priori }\end{array}$ & 5.87 & 0 \\
\cline { 2 - 4 } & $\begin{array}{c}\text { Constante } \\
2000 \mathrm{~m} / \mathrm{s}\end{array}$ & 5.87 & 0 \\
\cline { 2 - 4 } & $\begin{array}{c}\text { Crescente } \\
1500 \text { a } 6500 \mathrm{~m} / \mathrm{s}\end{array}$ & 5.85 & 0 \\
\hline \multirow{5}{*}{20} & $\begin{array}{c}\text { Sem } \\
\text { a priori }\end{array}$ & 9.22 & 0 \\
\cline { 2 - 4 } & $\begin{array}{c}\text { Constante } \\
2000 \mathrm{~m} / \mathrm{s}\end{array}$ & 9.22 & 0 \\
\cline { 2 - 4 } & $\begin{array}{c}\text { Crescente1500 a } \\
6500 \mathrm{~m} / \mathrm{s}\end{array}$ & 9.14 & 0 \\
\hline
\end{tabular}

Tabela 1 - Erros associados ao modelo de 1000 camadas pelo método SVD. $\epsilon_{\mathrm{V}}$ e $\epsilon_{\mathrm{d}}$ correspondem, respectivamente, ao erro dos modelo e dado.

\begin{tabular}{|c|c|c|c|}
\hline $\begin{array}{c}\text { Velocidades } \\
\text { RMS }\end{array}$ & A Priori & $\epsilon_{\mathrm{V}}(\%)$ & $\epsilon_{\mathrm{d}}(\%)$ \\
\hline \multirow{4}{*}{50} & $\begin{array}{c}\text { Constante } \\
2000 \mathrm{~m} / \mathrm{s}\end{array}$ & 5.87 & 0 \\
\cline { 2 - 4 } & $\begin{array}{c}\text { Crescente } \\
1500 \text { a } 6500 \mathrm{~m} / \mathrm{s}\end{array}$ & 5.85 & 0 \\
\hline \multirow{4}{*}{20} & $\begin{array}{c}\text { Constante } \\
2000 \mathrm{~m} / \mathrm{s}\end{array}$ & 9.23 & 0 \\
\cline { 2 - 4 } & $\begin{array}{c}\text { Crescente } \\
1500 \text { a } 6500 \mathrm{~m} / \mathrm{s}\end{array}$ & 9.12 & 0 \\
\hline
\end{tabular}

Tabela 2 - Erros associados ao modelo de 1000 camadas pelo método MRE. $\epsilon_{\mathrm{V}}$ e $\epsilon_{\mathrm{d}}$ correspondem, respectivamente, ao erro dos modelo e dado.

\section{Discussão e Conclusões}

A distribuição de velocidades intervalares obtida através da fórmula de Dix é igual a obtida pelo método SVD, ou seja, $\epsilon_{\mathrm{dix}}=\epsilon_{\mathrm{SVD}}$, para ambos os modelos (Figura 3) nas simulações sem informação a priori. É digno de nota que o erro dos parâmetros de dado foi nulo em todas as simulações $\left(\epsilon_{\mathrm{d}}=0\right)$.
As figuras residuais entre as velocidades intervalares verdadeiras e as obtidas pela fórmula de Dix, SVD e MRE mostram que os principais contrastes estão nos limites entre as camadas. E conforme esperado esse contraste aumenta quando diminui o número de velocidades RMS de 50 para 20, que representa o número de equações no problema inverso. Em teoria, esse efeito das interfaces na estimativa incorreta das velocidades pode ser minimizado com o aumento do número de velocidades RMS.

A utilização da fórmula de Dix para a obtenção de estimativas de campo de velocidades intervalares mostra-se uma abordagem limitada a modelos geológicos simples. Como o Pré-sal corresponde a um modelo geológico complexo, a inversão de velocidades intervalares foi uma alternativa válida para minimizar os erros na obtenção de estimativas das velocidades intervalares, sendo mais eficiente e robusta, apesar de apresentar maior custo computacional se comparada com a abordagem convencional.

Os melhores resultados foram com a utilização de informação a priori linearmente crescente vido à menor diferença entre esse a priori e as velocidades intervalares verdadeiras em relação ao outro. A aplicação do SVD com informação a priori ao problema de inversão de velocidades intervalares e a utilização do MRE mostraram-se úteis e eficazes na determinação de boas estimativas de velocidades intervalares para o modelo sintético bidimensional com sistemas subdeterminados.

Em resumo, a informação a priori tem um papel importante na qualidade dos resultados obtidos.

\section{Agradecimentos}

Os autores agradecem à FINEP pelo apoio à Rede Cooperativa de Pesquisa em Geofísica de Exploração (Rede 01 - Fases 3 e 5) e ao CNPq e PETROBRAS pelo apoio ao Instituto Nacional de Ciência e Tecnologia de Geofísica de Petróleo (INCT-GP). T. G. Santana agradece à ANP e à CAPES pela bolsa de mestrado. A. Bassrei agradece ao CNPq pelos projetos 307.427/20102 e 308.690/2013-3 (bolsa de produtividade de pesquisa),

\section{Referências}

Assine, M. L., Corrêa, F. S. e Chang, H. K., 2008. Migração de depocentros na Bacia de Santos: importância na exploração de hidrocarbonetos. Revista Brasileira de Geociências, 38: 111-127.

Menke, W., 1984. Geophysical Data Analysis, Discrete Inverse Theory: Academic Press, Orlando, Florida.

Ulrych, T., Bassrei, A., and Lane, M., 1990. Minimum relative entropy inversion of $1 \mathrm{D}$ with applications: Geophysical Prospecting, 38: 465-487. 